\title{
Assessing caffeine exposure in pregnant women
}

\author{
Sinead M. Boylan ${ }^{1}$, Janet E. Cade ${ }^{1}$, Sara F. L. Kirk ${ }^{1}$, Darren C. Greenwood ${ }^{1}$, Kay L. M. White ${ }^{1}$, \\ Susan Shires ${ }^{1}$, Nigel A. B. Simpson ${ }^{2}$, Chris P. Wild ${ }^{1}$ and Alastair W. M. Hay ${ }^{1}$ \\ ${ }^{1}$ Centre for Epidemiology and Biostatistics, University of Leeds, Leeds LS2 9LN, UK \\ ${ }^{2}$ Obstetrics and Gynaecology, D Floor, Clarendon Wing, Leeds General Infirmary, Leeds LS2 9NS, UK \\ (Received 30 March 2007 - Revised 6 December 2007 - Accepted 7 January 2008 - First published online 11 March 2008)
}

Studies on the effects of caffeine on health, while numerous, have produced inconsistent results. One of the most uncertain and controversial effects is on pregnancy outcome. Studies have produced conflicting results due to a number of methodological variations. The major challenge is the accurate assessment of caffeine intake. The aim of the present study was to explore different methods of assessing caffeine exposure in pregnant women. Twenty-four healthy pregnant women from the UK city of Leeds completed both a detailed questionnaire, the caffeine assessment tool (CAT) designed specifically to assess caffeine intake and a prospective $3 \mathrm{~d}$ food and drink diary. The women also provided nine saliva samples over two consecutive days for estimation of caffeine and a metabolite (paraxanthine). Caffeine intakes from the CAT and diary showed adequate agreement (intra-class correlation coefficient of 0.5). For saliva caffeine and paraxanthine measures, the between-sample variation (within the same woman) was greater than between-woman and between-day variation. However, there was still adequate agreement between these measures and the CAT. The CAT is a valuable tool that is now being used in a large prospective study investigating caffeine's role in pregnancy outcome.

Caffeine: Assessment: Pregnancy

Caffeine or 1,3,7-trimethylxanthine exhibits a wide range of physiological effects ${ }^{(1)}$, in particular, it is a stimulator of the central nervous system. It is rapidly absorbed from the gastrointestinal tract and crosses the placenta. Caffeine originates in the cocoa bean, tea leaf, mate leaf, kola nut, cocoa pod and guarana seeds, and is naturally found in coffee, tea, cola, cocoa, dietary supplements and herbal products. Smaller amounts are present in products derived from cocoa and chocolate- or coffee-flavoured desserts and it is also added to soft drinks, 'energy' drinks, and prescription and non-prescription drugs.

A particular interest with respect to caffeine is the possible effect on development in utero. An early study in mice showed increased congenital malformations in the offspring of mice when treated with caffeine ${ }^{(2)}$. More recent epidemiological studies have led to the conclusion that caffeine intakes during pregnancy $>300 \mathrm{mg} / \mathrm{d}$ carry an increased risk of both spontaneous miscarriage and low birth weight ${ }^{(3)}$. However, the epidemiological studies have been contradictory, in part due to different approaches to measuring caffeine intake. A number of studies have found a relationship between relatively high caffeine consumption and low birth weight ${ }^{(4-6)}$, whereas others suggest that caffeine does not have an adverse effect on birth weight ${ }^{(7-10)}$.

Differences between studies may have occurred for a number of reasons. Some did not consider changes in intake during pregnancy. Timing of caffeine assessment is important since many pregnant women reduce, or discontinue, caffeine intake during pregnancy, either consciously, or due to nausea or a heightened sense of smell. Other studies only measured caffeine intakes after pregnancy ${ }^{(6,9)}$. In addition, many investigations often used a limited approach to assess caffeine intake. Studies have used coffee consumption as a proxy for caffeine intake, or simply counting the number of drinks and averaging caffeine content according to beverage ${ }^{(11-13)}$. To date, no study of caffeine and pregnancy has made a comprehensive analysis of all food, drinks and drugs containing caffeine, nor has any study considered in detail the variation in caffeine content of individual portion sizes and brands of these foods and drinks. Misclassification of caffeine consumption makes it more difficult to identify health effects from caffeine and reduces comparability among studies.

Even if the caffeine content of different items of food and drink can be accurately identified, there remains the problem of accurate recall of intake. Biomarkers, such as caffeine and its metabolites in body fluids, may offer an adjunct to questionnaire-based assessments of intake. However, there are substantial inter-individual variations in caffeine metabolism which would be expected to influence the correlation between measured caffeine intake and plasma concentrations. In addition, during pregnancy the half-life of caffeine is increased. Despite this caveat, plasma concentrations of the caffeine metabolite paraxanthine and, to a lesser extent, 
caffeine itself did distinguish between different levels of caffeine intake in women during pregnancy ${ }^{(14)}$. Paraxanthine has both a longer half-life in blood than caffeine and concentrations vary throughout the day. Plasma paraxanthine is less sensitive to recent intake of caffeine and may therefore be a better marker of caffeine consumption than plasma caffeine. A less invasive approach, important during pregnancy or for studying children, may be to measure caffeine in saliva. Saliva drug concentrations are frequently related to unbound drug concentrations in plasma ${ }^{(15,16)}$ and caffeine intake has been correlated to both saliva caffeine and paraxanthine ${ }^{(17)}$.

The present study will inform a larger one investigating the role of caffeine in pregnancy outcome, in particular, low birth weight. The main study uses the caffeine assessment tool (CAT) at three time points to explore caffeine intake throughout pregnancy. The aim of the project reported here was to explore three different methods of assessing caffeine exposure in pregnant women during the first trimester only, using a CAT, a $3 \mathrm{~d}$ food and drink diary, and salivary concentrations of caffeine and paraxanthine.

\section{Materials and methods}

\section{Subjects and samples}

All pregnant women aged 18 years old or over, attending the antenatal clinic at Leeds General Infirmary (Leeds, UK) were eligible. Pregnant women younger than 18 years of age or who were receiving their maternity care elsewhere were not eligible to take part. Maternity records of women attending the clinic were checked for suitable women. Women were approached by the research assistant, informed about the study, and given an information sheet. Women who agreed to take part were given a background questionnaire, CAT, $3 \mathrm{~d}$ food and drink diary, and nine Salivettes ${ }^{\circledR}$ (Sarstedt Aktiengesellschaft and Co., Nümbrecht, Germany) to take home, complete and return. The study protocol was approved by the local research ethics committee and written informed consent was obtained from all subjects.

Using the CAT, the present study assessed caffeine intakes from weeks 5 to 12 of pregnancy only since this was the most appropriate time frame relative to the stage of pregnancy when most women attend the antenatal clinic for the first time, when women are called to the clinic at about 16 weeks of pregnancy. The food and drink diary was completed at the same time as the CAT although the time frames did not overlap. The diary asked about $3 \mathrm{~d}$ of intake at the time of completion whereas the CAT requested recall of intake during weeks 512 of pregnancy. While completing the food and drink diary, the women collected a total of nine saliva samples over two consecutive days. Women noted both the time of consumption of foods and drinks and of the saliva collection in the diary to ensure that the saliva measurements provided biomarkers of actual consumption for comparison with the recorded dose (food and drink diary). The saliva samples were also used to assess variation in salivary caffeine and paraxanthine concentrations at different times during the day, and between days. Each saliva sample was collected in a Salivette ${ }^{\circledR}$ (Sarstedt Aktiengesellschaft and Co.). The Salivette ${ }^{\circledR}$ consists of an outer centrifuge vessel containing a suspended insert and cotton wool swab. Women were required to keep the
Salivette ${ }^{\circledR}$ Swab in their mouth for 10 min to ensure adequate saliva collection. A sample interval of $90 \mathrm{~min}$ was chosen between collections to cause minimal disruption to normal daily activity. On the first day, each woman provided saliva samples every $90 \mathrm{~min}$ over a $9 \mathrm{~h}$ period, involving a total of seven saliva samples. To avoid the presence of caffeine in the saliva due to recent consumption (rather than following absorption), the women were asked to avoid caffeine-containing foods and drinks, listed on a sheet provided, for $1 \mathrm{~h}$ before collecting the first sample, and for 15 min before taking each of the following six samples. The women were also asked to rinse their mouth with tap water before collection. On the second day, the same women were asked to provide a further two saliva samples at approximately mid-morning and midafternoon to reflect likely time of sample collection in the larger study. Again the women were asked to avoid caffeine-containing foods and drinks for $1 \mathrm{~h}$ before sample collection and to rinse their mouth with tap water before the samples were taken. The women were told to refrigerate the samples until they were returned by post to the research team along with the background questionnaire, CAT and diary.

\section{Analysis of caffeine intakes}

The CAT was developed to assess caffeine intakes from all possible sources of caffeine in a FFQ style, taking into account specific brand, preparation and portion size information. Since the CAT was developed to assess caffeine intake during pregnancy, questions on consumption of caffeine-containing foods and beverages were repeated to assess intakes during different weeks of pregnancy. Brand information was collected on coffee, tea, hot chocolate, cola and energy drinks, and was categorised into types of drink, for example, instant, filter, iced, and place of consumption to remind women of beverage consumption outside the home or workplace. Further questions requested portion sizes of tea, coffee and cola, changes in intake of tea and coffee during pregnancy, methods of preparation, and intakes of foods which may affect caffeine metabolism, for example, cruciferous vegetables, grapefruit and barbecued foods ${ }^{(18-20)}$. The caffeine content of foods and beverages were obtained from a UK government report by the Ministry of Agriculture, Fisheries and Food ${ }^{(21)}$ and also from manufacturers and coffee-houses, providing caffeine values for twenty-nine instant coffees, nine filter coffees, three coffee-house filter coffees, a standard espresso shot and decaffeinated shot, eight instant beverage mixtures, seven espresso-based drinks, eighteen hot chocolates, twenty-five teas prepared from tea bags, seventeen teas prepared from tea leaves, one iced-tea, three instant teas, thirtysix colas, thirty-three energy drinks, eleven soft drinks and two alcoholic drinks. Each of the above beverages had a standard caffeinated and decaffeinated value assigned based on the average caffeinated and decaffeinated content of each drink. For each of the chocolate items a value was assigned based on the average caffeine content from various brands. The caffeine content of fifty-nine over-the-counter drugs was accessed from manufacturers' websites. The CAT also assesses possible confounders, for example, smoking habits, alcohol intake, use of medication and symptoms of pregnancy. The CAT is thus the most detailed and comprehensive tool to assess caffeine intake during pregnancy which is currently available. 
A detailed computer algorithm was developed which assigned a value for caffeine content $(\mathrm{mg})$ to all caffeinated products assessed in the CAT while taking into account portion sizes, brand information and frequency of intakes. Daily caffeine intake during weeks 5-12 of pregnancy could be calculated for each participant.

The food and drink diaries were analysed manually for each woman's daily caffeine intake. All caffeine-containing foods and drinks recorded in the diaries were allocated a caffeine value depending on amount and type of food or drink consumed. A mean caffeine value was calculated for each food and beverage source and assigned to any unbranded sources recorded in the diaries.

\section{Analysis of saliva}

Caffeine and metabolites were extracted and quantified using liquid:liquid extraction and reversed-phase HPLC with UV detection using a modification of the method of Butler et al. ${ }^{(22)}$. Salivettes ${ }^{\circledR}$ were thawed and saliva reclaimed by centrifugation at $756 \mathrm{~g}$ for $5 \mathrm{~min}$ at room temperature. Saliva $(180 \mu \mathrm{l})$ was added to a $5 \mathrm{ml}$ screw-cap tube containing $50 \mathrm{mg}$ ammonium sulfate following which $20 \mu \mathrm{l}$ of a stock solution of $\beta$-hydroxyethyltheophylline $(20 \mathrm{ug} / \mathrm{ml})$ was added as internal standard. The tube was shaken vigorously for $10 \mathrm{~s}$ using a Baxter multi tube vortex (Alpha Laboratories, Eastleigh, Hants, UK) on setting 4, to thoroughly mix the contents and precipitate any protein. This was followed by the addition of chloroform $(2.0 \mathrm{ml})$ and isopropanol $(0.5 \mathrm{ml})$ and the tube shaken again for $4 \mathrm{~min}$ on setting 4 . After centrifuging for $5 \mathrm{~min}(84 \mathrm{~g})$ the aqueous top layer was discarded and the organic layer transferred to a thick-walled glass tube and dried down under $\mathrm{N}_{2}$ at $45^{\circ} \mathrm{C}$. The residue was reconstituted in $30 \%$ methanol in water $(200 \mu 1)$, mixed vigorously for $3 \mathrm{~s}$ and centrifuged for $2 \mathrm{~min}(756 \mathrm{~g})$ to give a final concentration of $\beta$-hydroxyethyltheophylline of $2 \mu \mathrm{g} / \mathrm{ml}$. The reconstituted solution was transferred to an autosampler vial and $30 \mu \mathrm{l}$ injected onto a Beckman Ultrasphere column (ODS; $4.6 \mathrm{~mm} \times 25 \mathrm{~cm}$ ) with a short guard column $(5 \mathrm{~cm} \times 0.4 \mathrm{~cm})$ packed in-house with Bondapak C18/corasil (Waters, Elstree, Herts, UK). The solvent delivery system, autosampler and UV detector were manufactured by Gilson (Paris, France). UV wavelength was set at $280 \mathrm{~nm}$. Solvents used for elution were $0.045 \%$ acetic acid containing $9 \%$ methanol (A) and $100 \%$ methanol (B). Starting with solvent A, elution was a linear gradient over 5 min to a $2 \%$ solution of solvent $\mathrm{B}$, held at this for $10 \mathrm{~min}$, over the next $5 \mathrm{~min}$ increased in a linear gradient to $5 \%$ solvent $\mathrm{B}$, followed by a linear increase over $5 \mathrm{~min}$ to $8 \% \mathrm{~B}$, changed to $15 \% \mathrm{~B}$ and maintained for $15 \mathrm{~min}$, raised to $75 \% \mathrm{~B}$ and held at this for $10 \mathrm{~min}$, followed by reversion to $100 \% \mathrm{~A}$ (the starting solvent) which was maintained for $10 \mathrm{~min}$ to equilibrate the column before injection of the next sample. The flow rate was $1.2 \mathrm{ml} / \mathrm{min}$ and the retention time, in min, for each compound was approximately: theobromine, 10.3; 1,7-dimethyluric acid, 14.8; paraxanthine, 16.2 ; theophylline, $17.7 ; \beta-$ hydroxyethyltheophylline, 22.4; caffeine (1,3,7-trimethylxanthine), 29.5. Retention times varied slightly from day to day and were adjusted accordingly to produce optimum identification of each analyte when processing results. A single standard containing all the above compounds at the same concentration $(5 \mu \mathrm{g} / \mathrm{ml})$ was made up in $30 \%$ methanol; this was run after every five samples. Two 'in-house' qualitycontrol samples were also extracted and run with each batch of eighteen samples. Quality-control samples were made by spiking 'blanked saliva' with $5 \mu \mathrm{g} / \mathrm{ml}$ of each compound. The saliva, donated by volunteers, was collected in Salivettes ${ }^{\circledR}$ and 'blanked' by gentle mixing with charcoal $(0 \cdot 1 \mathrm{~g} /$ $\mathrm{ml})$ for $24 \mathrm{~h}$, centrifuged $(728 \mathrm{~g})$ for $10 \mathrm{~min}$ and the supernatant fraction filtered through a $0.20 \mu \mathrm{m}$ filter and stored at $-20^{\circ} \mathrm{C}$ until spiked. When in use, quality-control material was stored at $4^{\circ} \mathrm{C}$. Within-batch $\mathrm{CV}(\%)$ were: theobromine, 2.3; 1,7-dimethyluric acid, 2.4; paraxanthine, 2.2; theophylline, 2.5; $\beta$-hydroxyethyltheophylline, $3 \cdot 1 ; 1,3,7$-trimethylxanthine, 2.7. Between-batch CV (\%) were: theobromine, 2.8; 1,7-dimethyluric acid, 2.5; paraxanthine, 2.2; theophylline, $3.0 ; \beta$-hydroxyethyltheophylline, $2 \cdot 4 ; 1,3,7$-trimethylxanthine, $3 \cdot 8$. The limit of quantification was $50 \mathrm{ng} / \mathrm{ml}$ for all compounds, calculated from standards made up in $30 \%$ methanol with no extraction. The assay was linear between $50-10000 \mathrm{ng} / \mathrm{ml}$ for all the above compounds.

Caffeine, other methylxanthine metabolites and ammonium sulfate were purchased from Sigma (Poole, Dorset, UK) and all extraction and eluant reagents were supplied by Rathburn Chemicals (Walkerburn, Peebleshire, UK).

\section{Statistical analysis}

Statistical analysis was carried out using SPSS (version 10.1; SPSS Inc., Chicago, IL, USA), Stata (version 8.2; StataCorp LP, College Station, TX, USA) and MlwiN (University of Bristol, Bristol, UK) ${ }^{(23)}$. Caffeine intakes from the CAT and $3 \mathrm{~d}$ diary were compared. $\kappa$ Statistics were carried out to test the agreement between the caffeine intakes estimated either by the CAT or the diary (split at the median), and the mean of the seven saliva caffeine and paraxanthine measures on day 1 , and the mean of the two saliva caffeine and paraxanthine measures on day 2 . The total $3 \mathrm{~d}$ diary and the individual diary days which related to the day of saliva sample collection were used for this analysis. Time of gestation (weeks) was categorised into two groups (see Table 1 for categorisation), and agreement between the CAT and $3 \mathrm{~d}$ diary was estimated within each group as well as for the group as a whole. A variance components model was used to investigate the variance structure of the data and estimate the intraclass correlation coefficient (ICC) of agreement taking all sources of error into account.

\section{Results}

\section{Characteristics of women}

A total of sixty-three women were recruited, but only twentyfour completed the study. The mean age of the women was 31.7 (SD 3.4) years, with the mean gestation being 16.3 (SD 6.9) weeks. According to the Office for National Statistics socio-economic classification (SEC) self-coded method ${ }^{(24)}$ of calculating SEC status, the number of women classed as working in managerial and professional occupations, intermediate occupations, and as a small employer with account workers, were sixteen, five and one respectively. Two women did not fully complete the question on SEC status. 
Table 1. Caffeine intake (mg/d) and time of caffeine assessment tool (CAT) completion (Mean values and standard deviations)

\begin{tabular}{|c|c|c|c|c|c|c|c|c|c|}
\hline \multirow{2}{*}{ Tool } & \multicolumn{9}{|c|}{ Time of CAT and diary completion } \\
\hline & \multicolumn{3}{|c|}{ Total group ( $n$ 24) } & \multicolumn{3}{|c|}{$\leq 15$ weeks gestation $(n 13)$} & \multicolumn{3}{|c|}{$>15$ weeks gestation $(n 11)$} \\
\hline CAT & $128 \cdot 2$ & $129 \cdot 2$ & 0.50 & 123.4 & $90 \cdot 7$ & 0.69 & $133 \cdot 8$ & $168 \cdot 7$ & 0.29 \\
\hline Diary (mean intake over $3 d$ ) & $113 \cdot 2$ & $97 \cdot 0$ & & $98 \cdot 4$ & $91 \cdot 2$ & & $130 \cdot 1$ & $105 \cdot 0$ & \\
\hline
\end{tabular}

${ }^{*} \kappa$ Statistic comparing CAT and diary.

\section{Caffeine intakes}

The highest mean daily caffeine intakes calculated from the CAT and diary were among women who were of later gestation (Table 1). On average, the daily caffeine intake from the CAT at 128 (SD 129) $\mathrm{mg} / \mathrm{d}$ was $15 \mathrm{mg}$ more than the diary at 113 (SD 97) $\mathrm{mg} / \mathrm{d}$. This difference is small, being approximately one-fifth of a cup of instant coffee. The caffeine intakes from the CAT and the diary among the total sample ( $n$ 24) showed adequate agreement $(\mathrm{ICC}=0 \cdot 5)$. Ten of the women did not provide complete brand information on sources of caffeine intake in the diary despite doing so in the CAT. For these women, the mean daily caffeine intake from the CAT was 156 (SD 77) $\mathrm{mg} / \mathrm{d}$ which was $27 \mathrm{mg} / \mathrm{d}$ more than the diary at 129 (SD 80) $\mathrm{mg} / \mathrm{d}$. However, for the women who did provide complete brand information in the diary, the mean daily caffeine intake from the CAT was 108 (SD 156) $\mathrm{mg} / \mathrm{d}$; this was only $6 \mathrm{mg} / \mathrm{d}$ more than caffeine intake assessed from the diary at 102 (SD 109) $\mathrm{mg} / \mathrm{d}$. It is also evident that these women had lower caffeine intakes from both the CAT and diary than those who did not provide complete brand information in the diaries. Figure 1 illustrates the agreement between mean caffeine intake $(\mathrm{mg} / \mathrm{d})$ from the CAT and diary. The level of agreement between the two methods was greater for women who were $\leq 15$ weeks gestation (ICC $=0.69$ ) compared with those between 16 and 37 weeks gestation $(\mathrm{ICC}=0.29)$ (Table 1$)$. A greater level of agreement was also apparent when women who left education

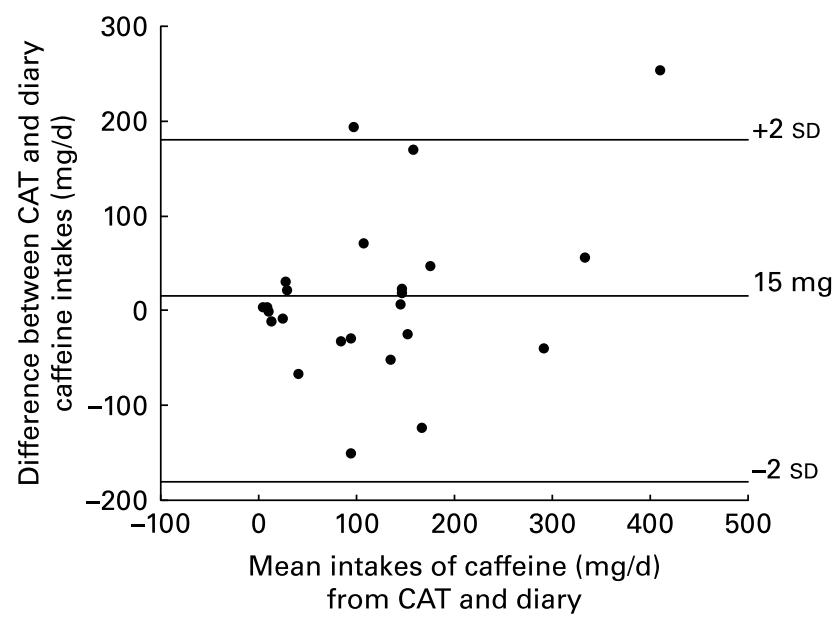

Fig. 1. Bland-Altman scatter plot of difference in caffeine intakes $(\mathrm{mg} / \mathrm{d})$ between the caffeine assessment tool (CAT) and food and drink diary. earliest were considered ( $\mathrm{ICC}=0.69$ ) compared with those who left education later (ICC $=0 \cdot 20$ ). Level of agreement between the two methods was similar for both young and old women (ICC $=0.50$ and 0.46 , respectively).

\section{Variability of saliva measures}

All nine saliva samples were available from sixteen women. The mean saliva caffeine and paraxanthine concentrations were 454 (95\% CI 367, 561) and 198 (95\% CI 165, 237) $\mathrm{ng} / \mathrm{ml}$ respectively. There was good agreement between days 1 and 2 of saliva collection for both saliva caffeine and paraxanthine (ICC $=0.66$ and 0.65 , respectively). Figure 2 illustrates the saliva sample concentrations and caffeine intake over the first day of saliva collection. It is evident from Fig. 2 that saliva caffeine and paraxanthine concentrations reflected each other closely for most women, for example, Fig. 2(a, b, f, j, k, u); however, this was not the case for a few of the women, for example, Fig. 2(i, p). It is also evident from Fig. 2 that salivary caffeine and paraxanthine concentrations reflected caffeine intake for some of the women, for example, Fig. 2(a, g, h, m). For some women, irrespective of level of caffeine intake, there was a sudden increase in saliva concentrations after caffeine intake, for example, Fig. 2(a, g, v). For others, however, saliva concentrations did not parallel caffeine intake, for example, Fig. 2(b, o), or had a delay in the development of peaks, for example, Fig. 2(h). Caffeine intakes were low for some women; therefore, peaks in saliva caffeine and paraxanthine concentrations were not as marked, for example, Fig. 2(i, r).

For both saliva caffeine and paraxanthine, the betweensample (i.e. within the same woman) variation was 50 and $61 \%$ of total variation, respectively, for example, Fig. 2(k) which shows considerable variation in saliva caffeine and paraxanthine between samples. Between-women variation for salivary caffeine and paraxanthine was 39 and $38 \%$ of total variation, respectively. Figure $2(\mathrm{~b}, \mathrm{k})$ shows how variable these concentrations are between women. Between-day variation for saliva caffeine and paraxanthine was relatively low at 11 and $0.1 \%$ of total variation, respectively. Despite this variation, however, the CAT agreed with the saliva measures just as well as with the food and drink diary. Using the $\kappa$ statistic, there was a moderate agreement between the CAT and saliva caffeine collected on both days 1 and 2 (0.50 and $0 \cdot 47$, respectively), with an even better agreement between the CAT and saliva paraxanthine collected on day $2(0 \cdot 65)$. Using the mean caffeine intake calculated over $3 \mathrm{~d}$ from the diary, a moderate agreement existed between the diary and 


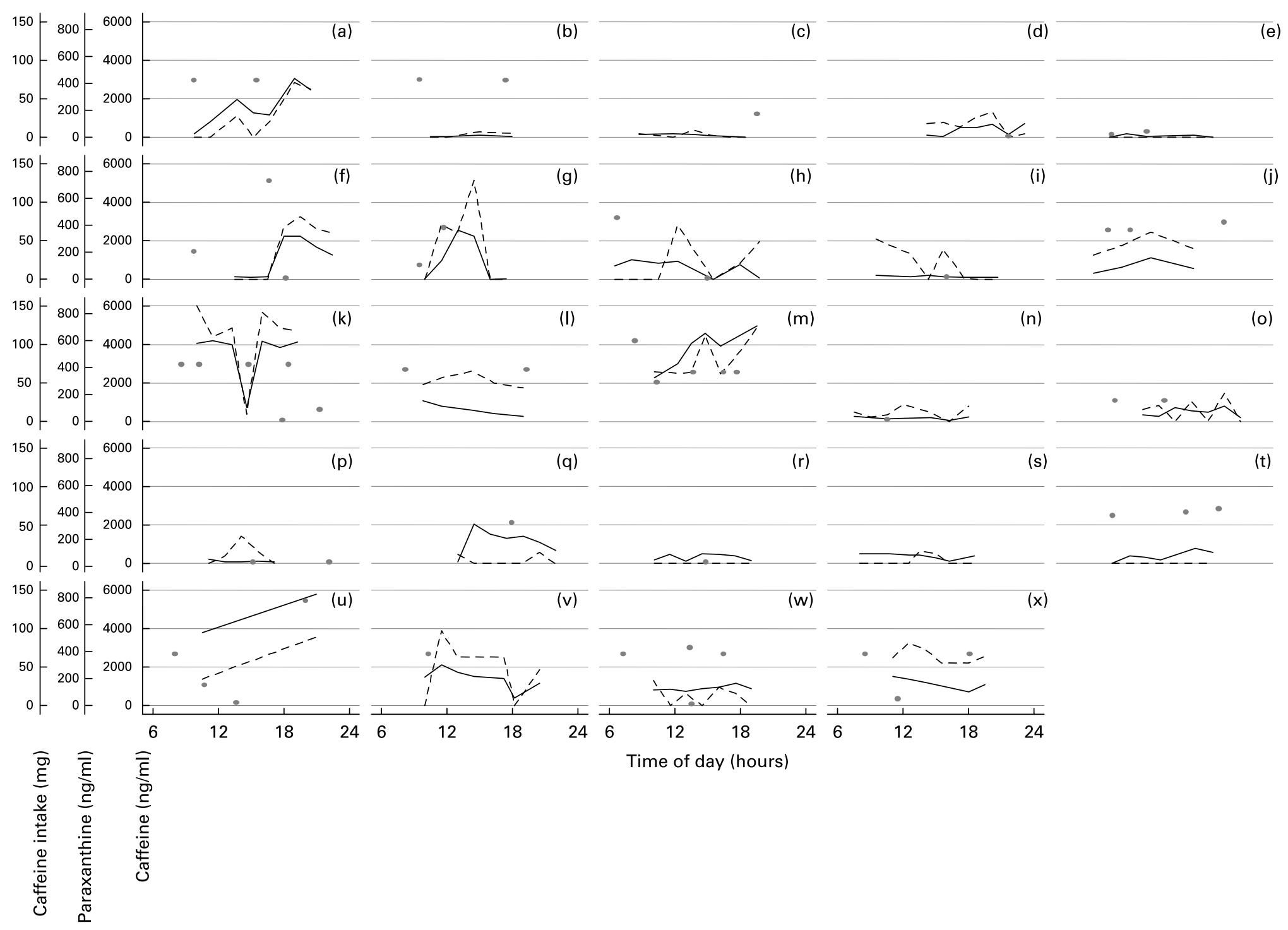

Fig. 2. Saliva measures of caffeine $(\mathrm{ng} / \mathrm{ml} ;-)$ and paraxanthine $(\mathrm{ng} / \mathrm{ml} ;---)$ collected on day 1 and caffeine intake $(\mathrm{mg} ; \mathrm{O})$ on the same day for each woman (a to $\mathrm{x})$. 
saliva paraxanthine collected on both day 1 and 2 (0.50 and 0.47 , respectively), with a greater agreement between the diary and saliva caffeine collected on day 1 (0.66). As expected, this agreement was even better when saliva concentrations were compared with caffeine intake from the diary on the same day of sample collection (Table 2).

\section{Discussion}

\section{Caffeine intakes}

The mean caffeine intakes from both the CAT and diary were lower than intakes previously reported by pregnant women in the UK $(204 \mathrm{mg} / \mathrm{d} \text { for a } 60 \mathrm{~kg} \text { woman })^{(25)}$. However, since this previous study, a report has been published by the Food Standards Agency (FSA) advising pregnant women to limit caffeine consumption to less than $300 \mathrm{mg} / \mathrm{d}^{(3)}$, and this may have decreased pregnant women's caffeine intakes in the UK. It is also possible that some women may have decreased or under-reported their caffeine intake as a result of taking part in the present study.

The CAT is a detailed assessment of caffeine intake which is straightforward to complete. In contrast, ten women did not provide detailed information on caffeine intake particularly relating to brand-level information in the diary even though they were instructed to do so. This may have contributed to the overall difference in estimated caffeine intakes between the CAT and the diary. There was a greater difference between caffeine intakes from the CAT and diaries from women who did not provide complete brand information compared with women who did provide this level of information in the diaries. Another contributor to difference in estimates of caffeine intakes between the two methods could be the different time periods assessed by the two tools. The diary assessed food and drink consumption over the $3 \mathrm{~d}$ whereas the CAT assessed recalled intakes over an 8 -week period. Despite the difference in estimates, there was still an adequate agreement between the two methods.

The level of agreement between the caffeine intakes from the CAT and diary was greatest among women who were in their earliest gestational weeks. This may be because the CAT that was administered in this test assessed caffeine intake early in pregnancy (weeks 5-12). Women later in pregnancy reported higher caffeine intakes in the CAT (Table 1), although only four women were in the second half of pregnancy (over 20 weeks); nevertheless, recall bias may have been introduced as these women may have been

Table 2. Agreement between caffeine intakes and saliva caffeine and paraxanthine measures

\begin{tabular}{lccc}
\hline & Day of sample & \multicolumn{2}{c}{$\begin{array}{c}\text { Coefficient of } \\
\text { agreement }(\kappa)\end{array}$} \\
\cline { 3 - 4 } Tool & 1 & Caffection & Paraxanthine \\
\hline Caffeine assessment & 2 & 0.50 & 0.33 \\
tool & 1 & 0.47 & 0.65 \\
Diary (intake on day & 2 & 0.74 & 0.57 \\
of saliva collection) & 1 & 0.45 & 0.64 \\
Total of 3d of diary & 2 & 0.67 & 0.50 \\
& & 0.30 & 0.48 \\
\hline
\end{tabular}

reporting caffeine intakes similar to their current intakes rather than intakes between weeks 5 and 12 of pregnancy. This is plausible, as caffeine intake may be lower in the first trimester due to nausea or intentional avoidance.

\section{Saliva samples}

In general, salivary caffeine and paraxanthine concentrations agreed with and reflected each other closely. It is also clear that the saliva concentrations generally responded to the caffeine intake recorded. However, for some of the women, the saliva measures did not appear to increase after reported caffeine intake. This may be due to error in completing the diary, or due to differences in metabolism and clearance between women resulting in lower concentrations at the time samples were collected. Caffeine is metabolised by the cytochrome P450 family of enzymes in the liver, in particular the major enzyme being cytochrome P450 1A2 (CYP1A2) ${ }^{(26)}$ with metabolites produced through demethylation and oxidation. Paraxanthine is a primary metabolite produced by demethylation of the $N^{3}$ position methyl group. Caffeine has a half-life of about $3-7 \mathrm{~h}$ unless the rate of action is affected by genetic and/or environmental factors, for example, CYP1A2 activity is decreased by female sex hormones during pregnancy or treatment with oral contraceptives ${ }^{(27)}$. There is wide variation in CYP1A2 mRNA expression; up to 40-fold variation has been described $^{(28,29)}$. Genetic polymorphisms of the CYP1A2 gene, smoking, chargrilled foods, brassica vegetables and prescription medicines also affect the rate of caffeine metabolism ${ }^{(30)}$. Saliva measures were chosen in preference to blood and urine due in part to the ease of obtaining the samples and the lower invasiveness of the measure for the subjects. Plasma and saliva clearance levels of caffeine are highly correlated ${ }^{(31)}$. Newton et al. ${ }^{(32)}$ concluded that salivary caffeine levels probably reflect the unbound plasma caffeine concentration and therefore can be used to estimate the pharmacokinetic parameters of the drug. They estimated that the overall saliva:plasma concentration ratio was 0.74 (SD 0.08). Other evidence has suggested that the complex metabolism of caffeine, together with different parameters controlling the renal clearance of each metabolite, makes the use of urinary metabolic ratios an inaccurate probe in populations ${ }^{(33)}$.

For the saliva measures, the between-sample variation was greater than the between-woman and between-day variation. This is expected, as the serum and saliva concentration of caffeine varies widely in response to recent caffeine intake. However, because of its longer half-life, paraxanthine concentrations will fluctuate less throughout the day and may be a better measure of caffeine intake. However, in the present study, the between-sample variation of saliva caffeine was lower than the between-sample variation of saliva paraxanthine. Despite this relatively large variability, Table 2 shows that, in general, both saliva measures adequately agreed with both the CAT and diary. As expected, the greatest level of agreement between the saliva measures and assessment of caffeine intake was found between seven saliva samples collected on day 1 and actual caffeine intake on that day, illustrating that a $1 \mathrm{~d}$ diary is a good snapshot of actual caffeine intake. Several measurements of salivary caffeine and paraxanthine over a day are far more likely to reflect intake than a single measurement. Both caffeine and paraxanthine have relatively short 
half-lives and concentrations in saliva (or plasma) change markedly over a day, reflecting recent consumption. Given that caffeine intake over the day is episodic, repeat measurements of salivary caffeine and paraxanthine are more likely to record this pattern of consumption than a single measurement at one time point.

A moderate, yet lower, agreement was found between the saliva measures and the more habitual intake calculated from the mean caffeine intake of the $3 \mathrm{~d}$ reported in the diary. Even though the CAT reflects longer-term habitual caffeine intake than the $3 \mathrm{~d}$ diary, the agreement between the CAT and saliva measures was only marginally less than that between the $3 \mathrm{~d}$ diary and saliva measures.

\section{General comments}

Despite using different methodologies, both the CAT and diary have emerged as equally good in assessing caffeine intake. However, it is the CAT that provides a practical, yet detailed, and therefore more accurate assessment of longterm habitual caffeine intake.

Only $38 \%$ of the women recruited took part in the present study, which could be due to the demands of completing a $3 \mathrm{~d}$ food and drink diary and collecting nine saliva samples and monitoring caffeine intake over $2 \mathrm{~d}$. It is important to consider that this sample of women may not be representative of the total pregnant population, as approximately one-third of the sample were employed in managerial and professional occupations.

As is evident from the present study, assessing long-term caffeine intake using food and drink diaries is not only impractical, but it is also likely to omit detail such as brand information. In the present study it was apparent that when such information was omitted from diaries, estimated caffeine intakes were on average $27 \mathrm{mg} / \mathrm{d}$ lower than intakes from the more detailed CAT - which is approximately equivalent to half a cup of tea. This suggests that the use of average values for sources of caffeine intake may underestimate caffeine intakes. A further source of error could be introduced by not considering strength of tea or coffee as commonly consumed. Different preparatory approaches to making tea or coffee can lead to variations in caffeine content ${ }^{(34)}$. We did ask women to record (in the CAT) the strength of tea and coffee they prepared and to state whether it was weak, medium or strong. We did not use this information, however, since there was limited published information available on variation in caffeine content by brand and preparation method. For instant coffee we did record and use in our analysis whether level or heaped teaspoons of dry coffee were used. Our previous experience of assessing diet has indicated that individual perceptions will vary and thus we could introduce more measurement error by using more subjective records. In addition, brewing times for cafetière-prepared coffee makes little difference to caffeine content of the brewed drink (PK Kadja, personal communication).

Repeated saliva measures may also be a useful measure of caffeine exposure. However, even if caffeine intake was accurately assessed, there exist inter-individual differences in metabolism that will influence spot saliva measurements. This may be especially relevant when assessing effects of caffeine exposure on pregnancy outcome, as caffeine metabolism decreases throughout pregnancy ${ }^{(35)}$.

A study is now being undertaken to assess the role of caffeine on pregnancy outcome, in particular low birth weight. Using the CAT, saliva measures of caffeine and metabolites, and a more detailed exploration of the inter-individual variations in caffeine metabolism, it will be possible to elucidate any links between caffeine and pregnancy outcome.

\section{Acknowledgements}

The present study was funded by the Food Standards Agency (FSA).

S. M. B. developed the project, carried out the fieldwork, undertook analysis and wrote the first draft of the paper. J. E. C. supervised the work and wrote the final draft of the paper. S. F. L. K. managed the project and commented on drafts of the paper. D. C. G. undertook some of the analysis and commented on drafts of the paper. K. L. M. W. developed some of the laboratory methods, undertook laboratory analysis and contributed to the paper. S. S. worked on the laboratory methods and undertook laboratory analysis. N. A. B. S. provided access to subjects and contributed to the design of the main study. C. P. W. supported the laboratory team and contributed to the design of the main study. A. W. M. H. developed laboratory methods, wrote some of the manuscript and supported the design of the study.

There is no conflict of interest.

\section{References}

1. Durrant KL (2002) Known and hidden sources of caffeine in drug, food, and natural products. $J$ Am Pharm Assoc 42, $625-637$.

2. Nishimura H \& Nakai K (1960) Congenital malformations in offspring of mice treated with caffeine. Proc Soc Exp Biol Med 104, 140-142.

3. Committee on Toxicity of Chemicals in Foods, Consumer Products and the Environment (COT) (2001) Statement on the Reproductive Effects of Caffeine. London: Food Standards Agency.

4. Beaulac-Baillargeon L \& Desrosiers C (1987) Caffeine-cigarette interaction on fetal growth. Am $J$ Obstet Gynecol 157, 1236-1240.

5. Martin TR \& Bracken MB (1987) The association between low birth weight and caffeine consumption during pregnancy. Am J Epidemiol 126, 813-821.

6. Vlajinac HD, Petrovic RR, Marinkovic JM, Sipetic SB \& Adanja BJ (1997) Effect of caffeine intake during pregnancy on birth weight. Am J Epidemiol 145, 335-338.

7. Barr HM \& Streissguth AP (1991) Caffeine use during pregnancy and child outcome: a 7-year prospective study. Neurotoxicol Teratol 13, 441-448.

8. Godel JC, Pabst HF, Hodges PE, Johnson KE, Froese GJ \& Joffres MR (1992) Smoking and caffeine and alcohol intake during pregnancy in a northern population: effect on fetal growth. CMAJ 147, 181-188.

9. Santos IS, Victora CG, Huttly S \& Carvalhal JB (1998) Caffeine intake and low birth weight: a population-based case-control study. Am J Epidemiol 147, 620-627.

10. Clausson B, Granath F, Ekbom A, Lundgren S, Nordmark A, Signorello LB \& Cnattingius S (2002) Effect of caffeine 
exposure during pregnancy on birth weight and gestational age. Am J Epidemiol 155, 429-436.

11. Linn S, Schoenbaum SC, Monson RR, Rosner B, Stubblefield PG \& Ryan KJ (1982) No association between coffee consumption and adverse outcomes of pregnancy. N Engl J Med 306, 141-145.

12. Mau G \& Netter P (1974) Are coffee and alcohol consumption risk factors in pregnancy? (article in German). Geburtshilfe Frauenheilkd 34, 1018-1022.

13. McDonald AD, Armstrong BG \& Sloan M (1992) Cigarette, alcohol, and coffee consumption and prematurity. Am J Public Health 82, 87-90.

14. Klebanoff MA, Levine RJ, DerSimonian R, Clemens JD \& Wilkins DG (1998) Serum caffeine and paraxanthine as markers for reported caffeine intake in pregnancy. Ann Epidemiol 8, $107-111$.

15. Danhof M \& Breimer DD (1978) Therapeutic drug monitoring in saliva. Clin Pharmacokinet 3, 39-57.

16. Horning MG, Brown L, Nowlin J, Lertratanangkoon K, Kellaway P \& Zion TE (1977) Use of saliva in therapeutic drug monitoring. Clin Chem 23, 157-164.

17. James JE, Bruce MS, Lader MH \& Scott NR (1989) Self-report reliability and symptomatology of habitual caffeine consumption. Br J Clin Pharmacol 27, 507-514.

18. Murray S, Lake BG, Gray S, Edwards AJ, Springall C, Bowey EA, Williamson G, Boobis AR \& Gooderham NJ (2001) Effect of cruciferous vegetable consumption on heterocyclic aromatic amine metabolism in man. Carcinogenesis 22, 1413-1420.

19. Fuhr U, Klittich K \& Staib AH (1993) Inhibitory effect of grapefruit juice and its bitter principal, naringenin, on CYP1A2 dependent metabolism of caffeine in man. Br J Clin Pharmacol 35, 431-436.

20. Fontana RJ, Lown KS, Paine MF, Fortlage L, Santella RM, Felton JS, Knize MG, Greenberg A \& Watkins PB (1999) Effects of a chargrilled meat diet on expression of CYP3A, CYP1A, and P-glycoprotein levels in healthy volunteers. Gastroenterology 117, 89-98.

21. Ministry of Agriculture, Fisheries and Food - Food Safety Directorate (1998) Survey of Caffeine and Other Methylxanthines in Energy Drinks and Other Caffeine-Containing Products (updated). 144. Food Survellance Information Sheet. London: MAFF.

22. Butler MA, Lang NP, Young JF, Caporaso NE, Vineis P, Hayes RB, Teitel CH, Massengill JP, Lawsen MF \& Kadlubar FF (1992) Determination of CYP1A2 and NAT2 phenotypes in human populations by analysis of caffeine urinary metabolites. Pharmacogenetics 2, 116-127.
23. Rasbash J, Browne W \& Goldstein H (2001) A User's Guide to MLwiN. London: University of London.

24. Office for National Statistics (2002) The National Statistics Socio-Economic Classification User Manual, 1st ed. London: HM Stationery Office.

25. Barone JJ \& Roberts HR (1996) Caffeine consumption. Food Chem Toxicol 34, 119-129.

26. Butler MA, Iwasaki M, Guengerich FP \& Kadlubar FF (1989) Human cytochrome P-450PA (P-4501A2), the phenacetin $O$-deethylase, is primarily responsible for the hepatic 3-demethylation of caffeine and $\mathrm{N}$-oxidation of carcinogenic arylamines. Proc Natl Acad Sci U S A 86, 7696-7700.

27. Zaigler M, Rietbrock S, Szymanski J, Dericks-Tan JSE, Staib AH \& Fuhr U (2000) Variation of CYP1A2-dependent caffeine metabolism during menstrual cycle in healthy women. Int J Clin Pharmacol Ther 38, 235-244.

28. Andersen MR, Farin FM \& Omiecinski CJ (1998) Quantification of multiple human cytochrome P450 mRNA molecules using competitive reverse transcriptase-PCR. DNA Cell Biol 17, 231-238

29. Finnstrom N, Thorn M, Loof L \& Rane A (2001) Independent patterns of cytochrome $\mathrm{P} 450$ gene expression in liver and blood in patients with suspected liver disease. Eur J Clin Pharmacol 57, 403-409.

30. Vineis P, Malats N, Lang M, d'Errico A, Caporaso N, Cuzick J \& Boffetta P (1999) Metabolic Polymorphisms and Susceptibility to Cancer. Lyon: International Agency for Research on Cancer.

31. Carrillo JA, Christensen M, Ramos SI, et al. (2000) Evaluation of caffeine as an in vivo probe for CYP1A2 using measurements in plasma, saliva, and urine. Ther Drug Monit 22, 409-417.

32. Newton R, Broughton LJ, Lind MJ, Morrison PJ, Rogers HJ \& Bradbrook ID (1981) Plasma and salivary pharmacokinetics of caffeine in man. Eur J Clin Pharmacol 21, 45-52.

33. Notarianni LJ, Oliver SE, Dobrocky P, Bennett PN \& Silverman BW (1995) Caffeine as a metabolic probe: a comparison of the metabolic ratios used to assess CYP1A2 activity. $\mathrm{Br} J \mathrm{Clin}$ Pharmacol 39, 65-69.

34. Bracken MB, Triche E, Grosso L, Hellenbrand K, Belanger K \& Leaderer BP (2002) Heterogeneity in assessing self-reports of caffeine exposure: implications for studies of health effects. Epidemiology 13, 165-171.

35. Aldridge A, Bailey J \& Neims AH (1981) The disposition of caffeine during and after pregnancy. Semin Perinatol 5, $310-314$. 